\title{
Ofloxacin-like Antibiotics Inhibit Pneumococcal Cell Wall-degrading Virulence Factors*
}

Received for publication, February 2, 2005, and in revised form, March 9, 2005 Published, JBC Papers in Press, March 15, 2005, DOI 10.1074/jbc.M501236200

\begin{abstract}
Carlos Fernández-Tornero $\$$, Ernesto Garcíaๆ, Beatriz de Pascual-Teresa |, Rubens Lópezๆ,

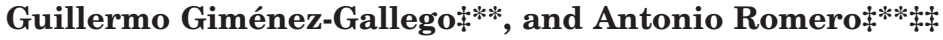

From the $\ddagger$ Departamento de Estructura y Función de Proteinas and $\uparrow$ Departamento de Microbiología Molecular, Centro de Investigaciones Biológicas, Consejo Superior de Investigaciones Científicas, C/Ramiro de Maeztu, 9, 28040 Madrid, Spain and the \|Departamento de CC Químicas, Facultad de Ciencias Experimentales y de la Salud, Universidad San Pablo CEU, Boadilla del Monte, 28668 Madrid, Spain
\end{abstract}

\begin{abstract}
The search for new drugs against Streptococcus pneumoniae (pneumococcus) is driven by the 1.5 million deaths it causes annually. Choline-binding proteins attach to the pneumococcal cell wall through domains that recognize choline moieties, and their involvement in pneumococcal virulence makes them potential targets for drug development. We have defined chemical criteria involved in the docking of small molecules from a three-dimensional structural library to the major pneumococcal autolysin (LytA) choline binding domain. These criteria were used to identify compounds that could interfere with the attachment of this protein to the cell wall, and several quinolones that fit this framework were found to inhibit the cell wall-degrading activity of LytA. Furthermore, these compounds produced similar effects on other enzymes with different catalytic activities but that contained a similar choline binding domain; that is, autolysin (LytC) and the phage lytic enzyme (Cpl-1). Finally, we resolved the crystal structure of the complex between the choline binding domain of LytA and ofloxacin at a resolution of $2.6 \AA$. These data constitute an important launch pad from which effective drugs to combat pneumococcal infections can be developed.
\end{abstract}

Streptococcus pneumoniae (pneumococcus) causes high morbidity and mortality worldwide through infections such as pneumonia, bacteremia, meningitis, acute otitis media, and sinusitis (1). The current scenario in which the vaccines available are not fully efficient and the resistance to antibiotics is ever-increasing makes the discovery of new drugs against this human pathogen rather urgent $(2,3)$. Among the determinants of pneumococcus virulence that have been identified (4), much attention is currently being focused on a family of surface-

* This work was supported in part by Ministerio de Educación y Ciencia (Spain) Grants BIO2001-1724 and BMC2003-00074. The costs of publication of this article were defrayed in part by the payment of page charges. This article must therefore be hereby marked "advertisement" in accordance with 18 U.S.C. Section 1734 solely to indicate this fact.

The atomic coordinates and structure factors (code 2BML) have been deposited in the Protein Data Bank, Research Collaboratory for Structural Bioinformatics, Rutgers University, New Brunswick, NJ (http: //www.rcsb.org/).

$\S$ Supported by a Ph.D. fellowship from the Ministerio de Educación y Ciencia. Present address: European Molecular Biology Laboratory, Grenoble Outstation, 6 rue Jules Horowitz, BP 181, 38042 Grenoble, France.

** Both authors contribute equally to this work.

t To whom correspondence should be addressed. Tel.: 34-91-8373112 (ext. 4244); Fax: 34-91-5360432; E-mail: romero@cib.csic.es. exposed polypeptides known as choline-binding proteins (ChBPs). ${ }^{1}$ The interest in these polypeptides has arisen because they are implicated in pathogenic processes such as adhesion to host cells, nasopharyngeal colonization, and bacterial sepsis (5). Although these enzymes contain different catalytic domains that account for a wide range of functions, they do all share a choline binding domain (ChBD) that consists of a series of $\sim 20$-amino acid-long imperfect choline binding repeats (6). ${ }^{2}$ The ChBD is responsible for the non-covalent anchoring of the ChBPs to the choline moieties of both teichoic and lipoteichoic acids at the cell surface (8). This peculiar attachment to the cell surface is essential for their contribution to bacterial virulence.

Three ChBPs catalyze the degradation of the pneumococcal murein when cell wall biosynthesis ceases, each one cleaving the peptidoglycan backbone at a different site (for review, see Ref. 9). Of these, the major autolysin (LytA) is an amidase (9), whereas the two minor peptidoglycan hydrolases (LytB and LytC) function as a glucosaminidase (10) and a lysozyme (11), respectively. LytA and LytC are responsible of the autolytic release of toxins like pneumolysin, which by irritating the host tissues allows pneumococcus to gain access to the bloodstream. It is in this way that the bacteria invade the lungs and the brain, causing bacteremia, meningitis, and pneumonia $(5,12)$.

In many cases the ChBPs modulate their own enzyme activity. Thus, in the case of LytA, the full-length protein obtained from ethanolamine-grown pneumococci or expressed in Escherichia coli remains in the inactive E-form until it is preincubated with $150 \mathrm{~mm}$ choline $(13,14)$. However, although the enzyme requires a high concentration of choline for its conversion to the active C-form, its activity remains largely undetectable unless the choline concentrations are lowered. This apparent paradox arises through the competition between the free amino alcohol and the choline moieties of the teichoic/lipoteichoic acids in the pneumococcal cell wall. The enzymatic activity of LytA depends on its binding to the cell wall, which is inhibited when it interacts with the free choline (15). Furthermore, in vivo experiments have shown that the sequestering of LytA by the lipoteichoic acids of the plasma membrane has a similar inhibitory effect (16). In addition, certain ChBPs such as LytA are irreversibly denatured in the absence of choline.

We recently described the crystal structure of the ChBD in LytA complexed with choline $(17,18)$. This dimeric structure reveals a novel solenoid fold consisting exclusively of $\beta$ hairpins that stack to form a left-handed superhelix. This superhelix is

\footnotetext{
${ }^{1}$ The abbreviations used are: ChBPs, choline-binding proteins; ChBD, choline-binding domain; ChBSs, choline-binding sites.

2 www.sanger.ac.uk/cgi-bin/Pfam/getacc?PF01473.
} 
Inhibition of Pneumococcal Virulence Factors

TABLE I

Autodock parameters used for flexible docking

Genetic algorithm (ga) and Lamarckian genetic algorithm parameters

ga_pop_size

ga_num_evals

ga_num_generations

ga_elitism

ga_mutation_rate

ga_crossover_rate

ga_window_size

ga_cauchy_alpha

ga_cauchy_beta

sw_max_its

sw_max_succ

sw_max_fail

sw_rho

sw_lb_rho

ls_search_freq
Number of individuals in population

Maximum number of energy evaluations

Maximum number of generations

Number of top individuals that automatically survive

Rate of gene mutation

Rate of crossover

Number of generations for picking worst individual

Mean of cauchy distribution for gene mutation

Variance of cauchy distribution for gene mutation Local search parameters

Number of iterations of Solis and Wets local search

Number of consecutive successes before changing rho

Number of consecutive failures before changing rho

Size of local search space to sample

Lower bound on rho

Probability of performing local search on an individual
50

250,000

27,000 stabilized by choline molecules at the hydrophobic interface of consecutive hairpins. Four choline-binding sites (ChBSs) are found per monomer, and all the forces contributing to the interaction between the protein and choline molecules are hydrophobic, with an electrostatic cation- $\pi$ contribution. We have used this crystal structure to screen a data base of small organic molecules to find new lead compounds that may diminish pneumococcal virulence by preventing the binding of $\mathrm{Ch}$ BPs to the cell wall. One such lead compound singled out in the screening procedure was ofloxacin, which mimics an inhibitor of its anchoring to the pneumococcal cell wall and of its enzymatic activity. With a view to providing a more precise basis for pursuing these leads, the crystal structure of the ChBD from LytA complexed to ofloxacin was resolved.

\section{EXPERIMENTAL PROCEDURES}

Purification of Lytic Enzymes-The biochemical characteristics of the pneumococcal autolysins LytA and LytC as well as those of the phage-coded lysines Cpl-1 and Cpl-7 have been recently reviewed (19). All these enzymes were overexpressed in $E$. coli and purified to electrophoretic homogeneity either by affinity chromatography on diethylaminoethanol (DEAE)-cellulose (LytA, LytC, and Cpl-1) $(20,21)$ or by using (NH4) $)_{2} \mathrm{SO}_{4}$ fractionation and gel filtration (22).

In Vitro Evaluation of the Inhibition of LytA Binding to the Cell Wall-An in vitro test based on the similarity between choline and DEAE was devised to evaluate the capacity of compounds to interfere with the binding of LytA to the cell wall. This similarity was used to devise an affinity chromatography-based purification protocol for this protein $(23,24)$. A sonicated extract from $E$. coli overexpressing either full-length LytA or its ChBD (17) was prepared in $50 \mathrm{~mm}$ Tris-HCl, $\mathrm{pH}$ $6.5,0.1 \mathrm{M} \mathrm{NaCl}$ buffer. This extract was centrifuged, and the supernatant was mixed with $\sim 2 \mathrm{ml}$ of DEAE-Sephacel (Amersham Biosciences) pre-equilibrated in the same buffer. The pellet recovered was then washed extensively with $50 \mathrm{~mm}$ Tris- $\mathrm{HCl}, \mathrm{pH} 6.5,1.5 \mathrm{M} \mathrm{NaCl}$ and divided in aliquots that were loaded into small chromatography columns. These columns were prepared using $0.5 \mathrm{ml}$ of bench-top centrifuge ultrafiltration cartridges of $0.45-\mu \mathrm{m}$ cutoff (Millipore), and they were eluted with $1 \mathrm{ml}$ of the sonication buffer containing the compound to be tested ( 0.5 to $25 \mathrm{~mm}$, depending on the solubility of the compound; Sigma-Aldrich). A solution containing $25 \mathrm{~mm}$ choline chloride was used as the positive control and the sonication buffer as a negative control. All steps were performed at $4{ }^{\circ} \mathrm{C}$. The presence of LytA (or its ChBD) in the eluted fractions was assessed by SDS-polyacrylamide gel electrophoresis (acrylamide 15\%; acrylamide/bisacrylamide ratio, 30:1).

Conversion Tests and Cell Wall Degradation Assays-For this assay we have adapted the protocol of García et al. (14). In brief, inactive LytA (E-form) overexpressed in $E$. coli was converted by incubating it at $4{ }^{\circ} \mathrm{C}$ with either 10 or $25 \mathrm{~mm}$ ofloxacin (Sigma). It was then subjected to the cell wall degradation assay as described previously (16). To evaluate the inhibition of cell wall degradation activity, the pure converted LytA amidase was tested in the presence of increasing concentrations of the different compounds under study (usually between 0.01 and $50 \mathrm{mM}$ ). As a control these reactions were performed with an equivalent concentration of choline chloride (Sigma), and all measurements were taken in quadruplicate. The same procedure was employed with LytC, Cpl-1, and Cpl-7 except that the $0.2 \mathrm{M}$ sodium phosphate buffer used was adjusted to $\mathrm{pH} 6.0$ rather than to 6.9 .

Structure-based Library Screening-The program AutoDock 3.0 (25) was chosen to analyze the computational docking of the compounds of the National Cancer Institute "Diversity" set data base with the ChBSs of LytA. For each ligand, hydrogen atoms and charges (Gasteiger) were added with SYBYL (SYBYL, Version 6.7, Tripos Inc., MO, www. tripos.com). The same program was used to add polar hydrogen atoms and Kollman united-atom partial atomic charges to the macromolecular structures. For the geometric representation of the macromolecular structure, the grids were approximately centered on the coordinates of the choline $\mathrm{N}$ atoms present in the crystal structure. The size of the grids $\left(22.5 \times 22.5 \times 22.5 \AA^{3}\right)$ was sufficient to include the entire ChBSs and provide enough space for random translation and rotational walking of the ligand across the surface. The grid spacing was taken to be $0.375 \AA$. The original Lennard-Jones and the hydrogen bonding potentials obtained using the Lamarckian genetic algorithm for docking were identical for all docking operations and are summarized in Table I.

Protein Preparation for Crystallization-To obtain crystals of the complex between the ChBD of LytA and ofloxacin, choline had to be removed from the protein sample. Therefore, the buffer used generally to elute the protein from the DEAE-Sephacel resin $(50 \mathrm{~mm}$ Tris-HCl, $\mathrm{pH}$ $6.5,0.1 \mathrm{M} \mathrm{NaCl}, 150 \mathrm{~mm}$ choline chloride) was switched to one in which choline was substituted with $5 \mathrm{~mm}$ ofloxacin (Sigma). The ChBD of LytA eluted under these conditions was stable in the absence of choline, which suggests that ofloxacin somehow mimics choline in stabilizing the general architecture of LytA. Protein/ofloxacin crystals were grown from a $12 \mathrm{mg} / \mathrm{ml}$ solution of the protein using the sitting drop vapor diffusion method at $295 \mathrm{~K}$ over a well solution of $2 \mathrm{M}$ ammonium sulfate and $2 \%(\mathrm{w} / \mathrm{v}$ ) polyethylene glycol 400 buffered with 0.1 м HEPES, $\mathrm{pH}$ 7.5. The typical crystal size was about $0.1 \times 0.1 \times 0.4 \mathrm{~mm}$.

Data Collection and Structure Determination-Diffraction data of single crystals were collected at $100 \mathrm{~K}$ with a MAR CCD detector with synchrotron radiation at the BM14S beamline from the European Synchrotron Radiation Facility (Grenoble, France). A single dataset was collected on a crystal diffracting to $2.6 \AA$. Data were processed with MOSFLM (26) and the CCP4 suite of programs (27). The crystals belonged to the $\mathrm{P} 2{ }_{1} 2{ }_{1} 2$ space group $(a=101.7 \AA, b=94.5 \AA, c=38.1 \AA$ ) with one dimer per asymmetric unit. The structure was determined by molecular replacement with $\mathrm{AMoRe}$ (28) using the coordinates of the ChBD of LytA complexed with choline (Protein Data Bank entry 1hcx) as a search model. Several cycles of simulated annealing and B-factor refinement were carried out using CNS (29) until the $R_{\text {work }}$ and $R_{\text {free }}$ values dropped to 22.0 and $28.0 \%$, respectively. Bulk solvent and anisotropic overall B factor corrections were applied throughout the refinement. Molecules at the ChBSs were only added to the densities evident in both the $2 F_{o}-F_{c}$ and the $F_{o}-F_{c}$ electron density maps after four refinement cycles. In the final model, which was validated using PROCHECK (30), no backbone $\varphi, \psi$ torsions angles were located in the disallowed region of the Ramachandran plot, whereas $83 \%$ of the residues were located in the most favored regions.

\section{RESULTS}

Docking of small $M_{r}$ Compounds into the ChBSs-We set out to identify lead compounds that might assist in the design of novel drugs to treat pneumococcus infections. To this end 


\begin{tabular}{|c|c|c|c|c|c|c|}
\hline \multirow{2}{*}{ NSC number ${ }^{1}$} & \multirow{2}{*}{ Chemical structure } & \multicolumn{5}{|c|}{ Lowest final docked energy $(\mathrm{kcal} / \mathrm{mol})^{2}$} \\
\hline & & ChBS1 & ChBS2 & ChBS3 & ChBS4 & Average \\
\hline 373529_2 & & -9.3 & -11.1 & -10.3 & -8.8 & $-9.9 \pm 1.1$ \\
\hline 361814 & & -10.0 & -8.2 & -10.8 & -8.7 & $-9.4 \pm 1.2$ \\
\hline 116490 & & -9.4 & -9.9 & -9.2 & -9.0 & $-9.4 \pm 0.4$ \\
\hline 600067 & & -9.6 & -8.8 & -10.0 & -9.0 & $-9.3 \pm 0.6$ \\
\hline 128609 & & -9.0 & -10.4 & -9.0 & -9.0 & $-9.3 \pm 0.7$ \\
\hline 116491 & & -9.1 & -9.9 & -9.2 & -8.8 & $-9.2 \pm 0.5$ \\
\hline 51535 & & -9.8 & -9.7 & -8.5 & -8.6 & $-9.1 \pm 0.7$ \\
\hline 3354 & & -8.9 & -9.8 & -9.0 & -8.7 & $-9.1 \pm 0.5$ \\
\hline 26645 & & -8.7 & -9.6 & -8.7 & -8.9 & $-9.0 \pm 0.4$ \\
\hline 76026 & & -9.4 & -8.7 & -9.5 & -8.1 & $-8.9 \pm 0.6$ \\
\hline 96021 & & -8.7 & -8.9 & -9.2 & -8.9 & $-8.9 \pm 0.2$ \\
\hline
\end{tabular}


TABLE II-continued

\begin{tabular}{|c|c|c|c|c|c|c|}
\hline \multirow{2}{*}{ NSC number ${ }^{1}$} & \multirow{2}{*}{ Chemical structure } & \multicolumn{5}{|c|}{ Lowest final docked energy $(\mathrm{kcal} / \mathrm{mol})^{2}$} \\
\hline & & ChBS1 & ChBS2 & ChBS3 & ChBS4 & Average \\
\hline 79050 & & -8.6 & -10.2 & -8.4 & -8.5 & $-8.9 \pm 0.8$ \\
\hline 18891 & & -8.6 & -9.0 & -8.4 & -9.5 & $-8.9 \pm 0.5$ \\
\hline 93241 & & -8.3 & -9.2 & -8.8 & -9.1 & $-8.8 \pm 0.4$ \\
\hline 371684 & & -9.3 & -8.8 & -8.7 & -8.5 & $-8.8 \pm 0.3$ \\
\hline 361815 & & -8.8 & -9.3 & -8.8 & -8.3 & $-8.8 \pm 0.4$ \\
\hline $11241 \_2$ & & -9.9 & -8.9 & -8.0 & -8.5 & $-8.8 \pm 0.8$ \\
\hline $11241 \_1$ & & -9.9 & -8.2 & -9.2 & -8.0 & $-8.8 \pm 0.9$ \\
\hline 127133 & & -9.7 & -8.1 & -9.0 & -8.3 & $-8.8 \pm 0.7$ \\
\hline 327705 & & -8.2 & -8.9 & -9.0 & -9.0 & $-8.8 \pm 0.4$ \\
\hline
\end{tabular}

${ }^{1} 129.43 .27 .140 /$ ncidb2

${ }^{2}$ The average values for choline and dextrofloxacin are $-3.8 \pm 0.4$ and $-6.0 \pm 0.6$, respectively.

molecular modeling studies were carried out using the crystal structure of the LytA ChBD to find small molecules that could block the interaction of this domain with the cell wall of pneumococcus. The program AutoDock 3.0 (25) has been successfully used to predict HIV-1 protease inhibitor binding (31) and the binding of haptens to antibodies (32). Thus, we used this software to analyze the docking process. The coordinates for the protein templates were those deposited in the Protein Data Bank after eliminating all the choline and water molecules (ID code $1 \mathrm{hcx}$ ). To reduce the total calculation time, we employed a pre-filtered collection of small molecules known as the Diversity set data base. This data base was derived from the almost 140,000 compounds available at the National Cancer Institute Developmental Therapeutics Program by selection of defined pharmacophores (33). The re- sulting list includes some 1,990 compounds that was further reduced by excluding those 167 ligands containing halogen or phosphor atoms because we found it difficult to deal with their energy calculation parameters.

AutoDock is designed to analyze the docking of small flexible ligands to a rigid protein. For this reason, different conformations of the four ChBSs of the monomeric ChBD cannot be explored using this program despite their high degree topological similarity. Consequently, four different docking operations for the 1823 compounds had to be performed. Choline was docked as a positive control, and in most cases, the final docked conformation was very similar to the one present in the crystal structure (not shown). The 20 best-score solutions yielded final docking energy values $\sim 2.5$ times higher than choline (Table II). 

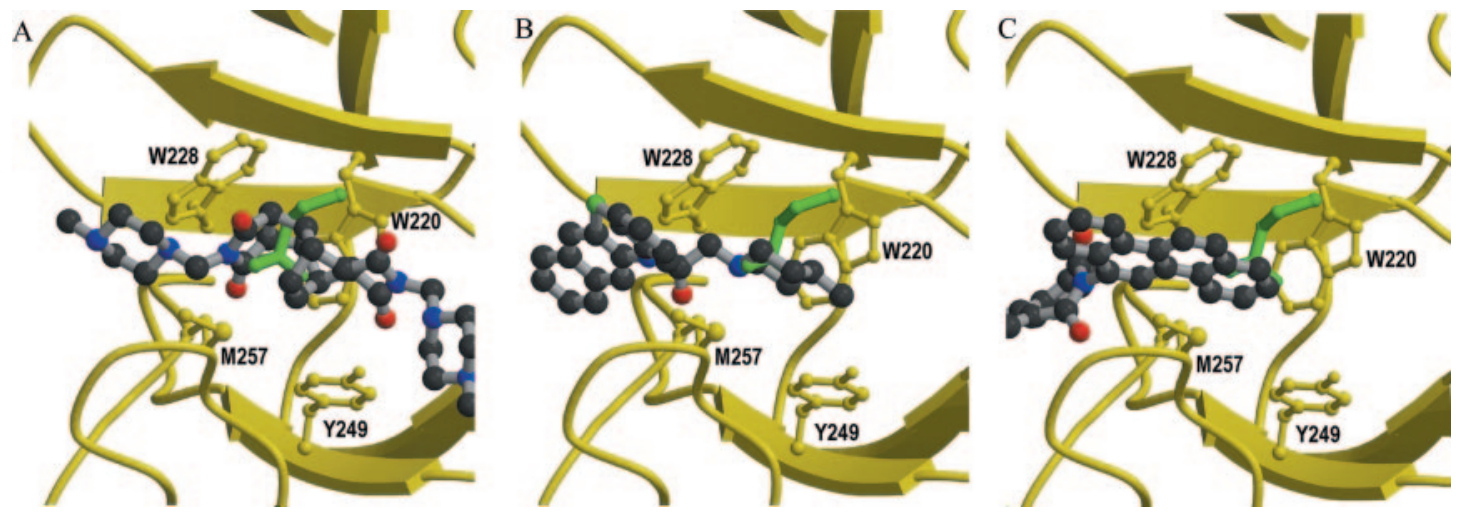

FIG. 1. Selected examples of the best-score docking solutions. The protein structure is represented in yellow at the back of the figure, with the aromatic residues of the ChBS as ball and stick. Compounds 373529_2 (A), $116490(B)$, and $128609(C)$ are docked onto the ChBS of the protein and are shown at the front as ball and stick, with the $\mathrm{C}, \mathrm{O}$, and $\mathrm{N}$ atoms colored black, red, and blue, respectively. In our crystal structure (green rods), choline has been included in the figure to illustrate the results of the docking.

The final docked conformations of the 20 best-score ligands were visualized using Turbo-Frodo (34). Although the threedimensional structures of these compounds are different, they seem to share certain characteristic structural features (Fig. 1). All the compounds contained a set of rings (almost invariably $6-8)$ that belong to the class of the planar or non-planar polycyclic structures. The small hydrophobic cavity that constitutes the ChBSs was always occupied by one or more of these rings. The planar rings generally bound in groups of $2-4$, indicating that the docking of individual planar rings into the ChBSs does not seem to be energetically favored. In contrast, the nonplanar structures bound through 1,3 , 4, or 5 rings, in which $\mathrm{N}$ or $\mathrm{O}$ atoms sometimes appear. They generally established hydrogen bonds with specific protein residues in the surroundings of the ChBSs.

Compounds Interacting with ChBSs under Physiological Conditions-Although the National Cancer Institute Diversity data base contains almost 2000 freely available compounds, these cannot be acquired in large enough quantities to carry out activity assays. Moreover, most of the best-score solutions obtained from the docking analysis are not soluble in aqueous solution, hampering the analysis of their activity under physiological conditions. As an alternative, we tested a set of commercially available compounds with a simple structure that retained most of the important structural features of the best docking compounds summarized above (Table III).

An initial estimate of the ability of compounds to interact with ChBSs and subsequently release LytA from the cell wall was carried out using DEAE-Sephacel (see "Experimental Procedures" and Table III). Only one of these compounds, ofloxacin, released LytA from the DEAE-Sephacel in a similar manner to choline (Fig. 2, lanes $S$ and $P$ ). This compound is a chiral molecule, commercially available as a racemic mixture of levoand dextrofloxacin. Elution was achieved at a concentration of $\sim 4 \mathrm{~mm}$, and it had a similar effect when either full-length LytA or its ChBD alone was bound to the resin (not shown). Furthermore, at a concentration of $25 \mathrm{~mm}$ ofloxacin, like free choline, was also able to convert LytA from the inactive E-form to the active C-form (not shown).

High Resolution Structure of C-LytA Bound to OfloxacinWe analyzed crystals of the complex between the LytA ChBD and ofloxacin, and the data processing and refinement statistics of a single crystal diffracting to $2.6 \AA$ are presented in Table IV. The corresponding structure represents a dimer of the LytA ChBD, which has the same characteristics as the previously reported crystal structures of the protein $(17,18)$. Polyethylene glycol molecules occupy most ChBSs, whereas only the ChBS2 of one monomer contains ofloxacin. As men- tioned, ofloxacin is a chiral molecule, and the enantiomer that best fitted the electron density maps was dextrofloxacin. Both the $2 F_{o}-F_{c}$ and $F_{o}-F_{c}$ Fourier maps clearly showed the presence of the dextrofloxacin molecule (Fig. $3 A$ ). The isolated non-planar methylpiperazine ring is completely inserted into the ChBS, probably mimicking the function of the hydrophobic head of choline (Fig. $3 B$ ). In contrast, the double-aromatic, planar system holding the negatively charged carboxylic group points toward the solvent. The methyl constituent of the piperazine ring points directly toward the Met residue in ChBS. The fluorine atom establishes a hydrogen bond with a water molecule, which is also hydrogen-bonded to the N $\epsilon$ atom of Trp-220 (Fig. 4A). It is important to note that the planar system partially interacts with the aromatic ring of a Tyr residue from a symmetric protein molecule. Docking of ofloxacin using the AutoDock program shows that this compound fit the ChBSs with a geometry reminiscent to that observed in the crystal structure (Fig. 4B). Although in the docked model, the dextrofloxacin molecule is positioned in a conformation slightly different from that observed in the crystal structure, the piperazine moiety fit into the ChBS, establishing similar hydrophobic interactions with the side chains of Trp-220 and Trp-228. Differences may be attributed to limitations of the energies parameters used by the AutoDock program.

Inhibition of Cell Wall Degradation by Ofloxacin and Related Compounds-The interaction of ofloxacin with the ChBSs shown in the high resolution structure would be expected to release LytA from the cell wall and, consequently, to inhibit its pneumococcal cell wall-degrading activity. This is indeed the case, and its inhibitory activity appeared to be similar to that of choline (Table V). Furthermore, as might have been expected from the sequence homology among ChBDs (35), this fluoroquinolone specifically inhibited other enzymes whose activity requires their attachment to the cell wall through ChBDs. Indeed, the minor pneumococcal lysozyme LytC and the Cpl-1 lysozyme encoded by the pneumococcal phage $\mathrm{Cp}-1$ are both inhibited by ofloxacin at concentrations below those required for choline (Table V). However, the choline-independent Cpl-7 lysozyme (35), which has a catalytic N-terminal domain virtually identical to that of Cpl-1 but lacks the ChBD, was not affected by ofloxacin even at the high concentrations (50 mM).

The piperazine ring of ofloxacin that interacts with the LytA ChBD is also present in norfloxacin, lomefloxacin, and pipemidic acid. Accordingly, the capacity of these three compounds to inhibit the cell wall-degrading activity of LytA was also tested. These compounds, with the exception of pipemidic acid, proved to be better inhibitors of the cell wall degrading activity of LytA. In our experiments, they showed an activity at least 
TABLE III

Compounds tested in vitro for ChBs binding

No. Compound name Basic formula Basic structure Comment

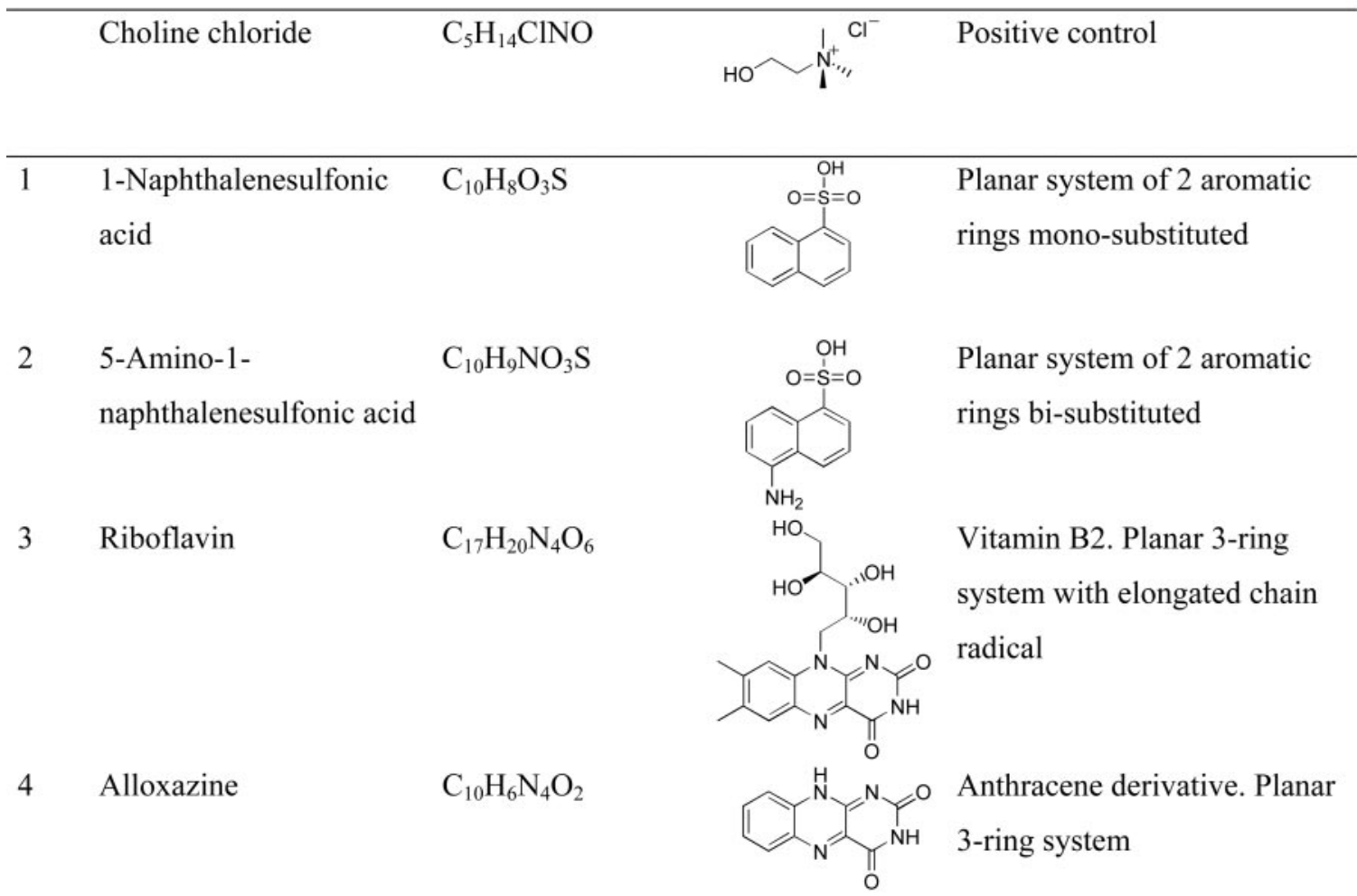

5 Lumichrome $\quad \mathrm{C}_{12} \mathrm{H}_{10} \mathrm{~N}_{4} \mathrm{O}_{2}$<smiles></smiles>

6 Ofloxacin $\quad \mathrm{C}_{18} \mathrm{H}_{20} \mathrm{FN}_{3} \mathrm{O}_{4}$

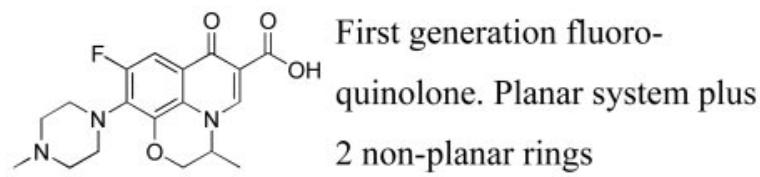

$7 \quad$ Norfloxacin

$\mathrm{C}_{16} \mathrm{H}_{18} \mathrm{FN}_{3} \mathrm{O}_{3}$<smiles>CCn1cc(C(=O)O)c(=O)c2cc(F)c(N3CCNCC3)cc21</smiles>

First generation fluoroquinolone. Planar 2-ring system plus non-planar ring

8 Lomefloxacin $\mathrm{C}_{17} \mathrm{H}_{19} \mathrm{~F}_{2} \mathrm{~N}_{3} \mathrm{O}_{3}$<smiles>CCn1cc(C(=O)O)c(=O)c2cc(F)c(N3CCNC(C)C3)c(F)c21</smiles>
Second generation fluoroquinolone. Planar 2-ring system plus non-planar ring

$9 \quad$ Pipemidic acid $\mathrm{C}_{14} \mathrm{H}_{17} \mathrm{~N}_{5} \mathrm{O}_{3}$<smiles>CCn1cc(C(=O)O)c(=O)c2cnc(N3CCNCC3)nc21</smiles>
Nalidixic acid derivative. Planar 2-ring system plus non-planar ring

one order of magnitude greater than both choline and ofloxacin (Table V).

Despite the structural similarities between ofloxacin and norfloxacin, lomefloxacin, or pipemidic acid (Table III), none of these three compounds was capable of releasing LytA protein from DEAE-Sephacel. This was unexpected since they inhibit its cell wall degrading activity and, thus, should all hinder the attachment of LytA to the cell wall (Table V). Similarly, attempts to crystallize these compounds bound to the ChBD of LytA also failed because the protein aggregated during the dialysis aimed to exchange choline from the affinity chromatography elution buffer. This could be attributed either to the 


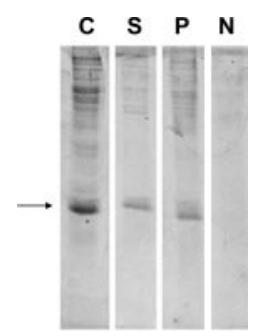

FIG. 2. Elution of LytA ChBD from DEAE-Sephacel minicolumns by ofloxacin. SDS-PAGE analysis of the protein eluted with 4 mM ofloxacin (lane $S$ ), $25 \mathrm{~mm}$ choline (lane $P$ ), and the vehicle (lane $N$ ). Lane $C$ corresponds to an $E$. coli crude extract of cells overexpressing the ChBD of LytA (arrow). Equivalent results were obtained with full-length LytA.

TABLE IV

Data collection, phasing, and refinement statistics

\begin{tabular}{lc}
\hline Data collection statistics & $\mathrm{P} 2{ }_{1} 2_{1} 2$ \\
Space group & $a$ \\
Cell dimensions $(\AA)$ & $201.7, b=38.1$ \\
No. molecules/asymmetric unit & 1.0163 \\
Wavelength $(\AA)$ & $40.0-2.6$ \\
Resolution $(\AA)$ & 107,594 \\
Measurements & 9,925 \\
Unique reflections & $6.3(27.2)$ \\
$R_{\text {sym }}(\%)^{a}$ & $8.6(2.6)$ \\
$I / \sigma^{\prime}(I)^{a}$ & $87.1(91.5)$ \\
Completeness $(\%)^{a}$ & \\
Structure determination & \\
statistics & 33.4 \\
Molecular replacement C-factor & 48.8 \\
Molecular replacement R-factor & \\
Refinement statistics & $6.0-2.6$ \\
Resolution range $(\AA)$ & $8,402 / 743$ \\
Reflections $(\text { work/free })^{b}$ & $22.0 / 28.0$ \\
$R_{\text {work }} / R_{\text {free }}(\%)$ & \\
Root mean square deviations & 0.008 \\
Bond lengths $(\AA)$ & 1.3 \\
Bond angles $\left({ }^{\circ}\right)$ & 24.6 \\
Dihedral angles $\left({ }^{\circ}\right)$ & \\
Number of atoms & 2,108 \\
Protein & 108 \\
Polyethylene glycol/ofloxacin & 56 \\
Solvent & 46.6 \\
Average B factor $\left(\AA^{2}\right)$ & \\
\hline &
\end{tabular}

${ }^{a}$ Values in parentheses correspond to the highest resolution shell $(2.74-2.60 \AA)$.

${ }^{b}$ Reflections in the test set represent $8 \%$ of the total number of reflections.

poor solubility of these compounds at the adequate $\mathrm{pH}$ values required for protein stability. Alternatively, they may be incapable of mimicking the stabilizing effect of choline on the ChBDs. Both these reasons may also explain why norfloxacin, lomefloxacin, and pipemidic acid fail to elute LytA from DEAESephacel. Nevertheless, their activity as specific inhibitors seems to suggest that they are able to effectively release LytA from its attachment to choline residues of the cell wall although they fail to stabilize the ChBD architecture in solution.

\section{DISCUSSION}

Although the virulence of pneumococcus is a major health concern, it is commonly accepted that the absence of ChBPs in the cell wall makes this bacteria remarkably less virulent $(5,36)$. For this reason, these proteins have attracted much attention as potential targets for drug development. Because cell wall attachment is essential for the enzymatic activity of ChBPs, forcing the release of these proteins from the cell wall with compounds that occupy the ChBSs might be an effective therapeutic approach (17). In addition, some of these enzymes can be activated by choline binding, and it might be possible to competitively impair this conversion $(13,14)$. Indeed, certain compounds might even promote the accumulation of the en- zyme in the inactive state by displacing choline. Finally, because choline seems to be important in stabilizing the threedimensional structure of certain $\operatorname{ChBPs}(17,18)$, it seems worthwhile exploring whether compounds that displace choline from the ChBSs may lack its stabilizing properties. These three therapeutic strategies are all obviously based on irreversibly displacing choline from its binding sites.

In the pursuit of chemicals that might give rise to the development of new drugs, efforts have been concentrated on two fronts, experimental high-throughput screening of compounds and virtual library searches using computational methods, a procedure known as structure-based drug design (37, 38). This latter procedure is faster and cheaper, but it requires that an atomic model of the target protein is available. The availability of the ChBD crystal structure from LytA at a resolution of $2.6 \AA$ (17) prompted us to search a library to identify compounds that might fit into the ChBS structure defined in this atomic model.

We initially performed a docking search of a three-dimensional structure data base containing almost 2000 compounds, which yielded a list of 20 putative ChBSs ligands. The interaction energies of these 20 ligands were at least twice that calculated for choline (Table II). These results must be considered as an approximation given the intrinsic problems of the currently available docking programs. For example, in general, flexible docking is only applied to the ligand and not to the macromolecule. Moreover, the scoring functions employed involve several simplifications and as such do not account for solvent molecules and some entropic contributions that may be essential for the correct estimation of protein-ligand affinity.

The compounds that were of most interest based on the docking calculations (Table II) are freely available from the National Cancer Institute but cannot be acquired in sufficiently large quantities for activity assays. Furthermore, most of them are not soluble in aqueous solution, which hampers their study in biologically relevant conditions. For these reasons the docking results could only be employed to establish a few rules to single out commercially available compounds whose interaction with ChBDs could be tested under physiological conditions.

Ofloxacin, a piperazine-containing fluoroquinolone, emerged as an active compound against the cell wall anchorage of pneumococcal LytA. We found that ofloxacin attaches to the ChBD of LytA through its piperazine moiety. Furthermore, it mimics choline in the enzyme conversion process, in the stabilization of the ChBD architecture, and in the inhibition of the protein cell wall degrading activity. We found that this inhibition is specifically mediated by the interaction of ofloxacin with the ChBD of LytA. Moreover, we demonstrate that other piperazine-containing quinolones, such as norfloxacin, lomefloxacin, and pipemidic acid, also inhibit the enzymatic activity of LytA.

The irreversible denaturalization of LytA in the dialysis experiments, aimed to exchange choline with either norfloxacin, lomefloxacin, or pipemidic acid, has no straightforward interpretation but could be a phenomenon of certain interest. It is well known that LytA is not stable in the absence of choline, a feature easily explained by the three-dimensional structure of its ChBD bound to choline (17). Therefore, the instability observed in the dialysis experiments could be explained purely on the basis of choline dilution. This does not occur when ofloxacin is included in the dialysis buffers since the threedimensional structure presented here shows that ofloxacin can assume the structural role of choline in stabilizing the ChBD of LytA. It is surprising that this stabilization does not occur with norfloxacin and lomefloxacin, given that they are considerably more efficient inhibitors of LytA activity than choline or 

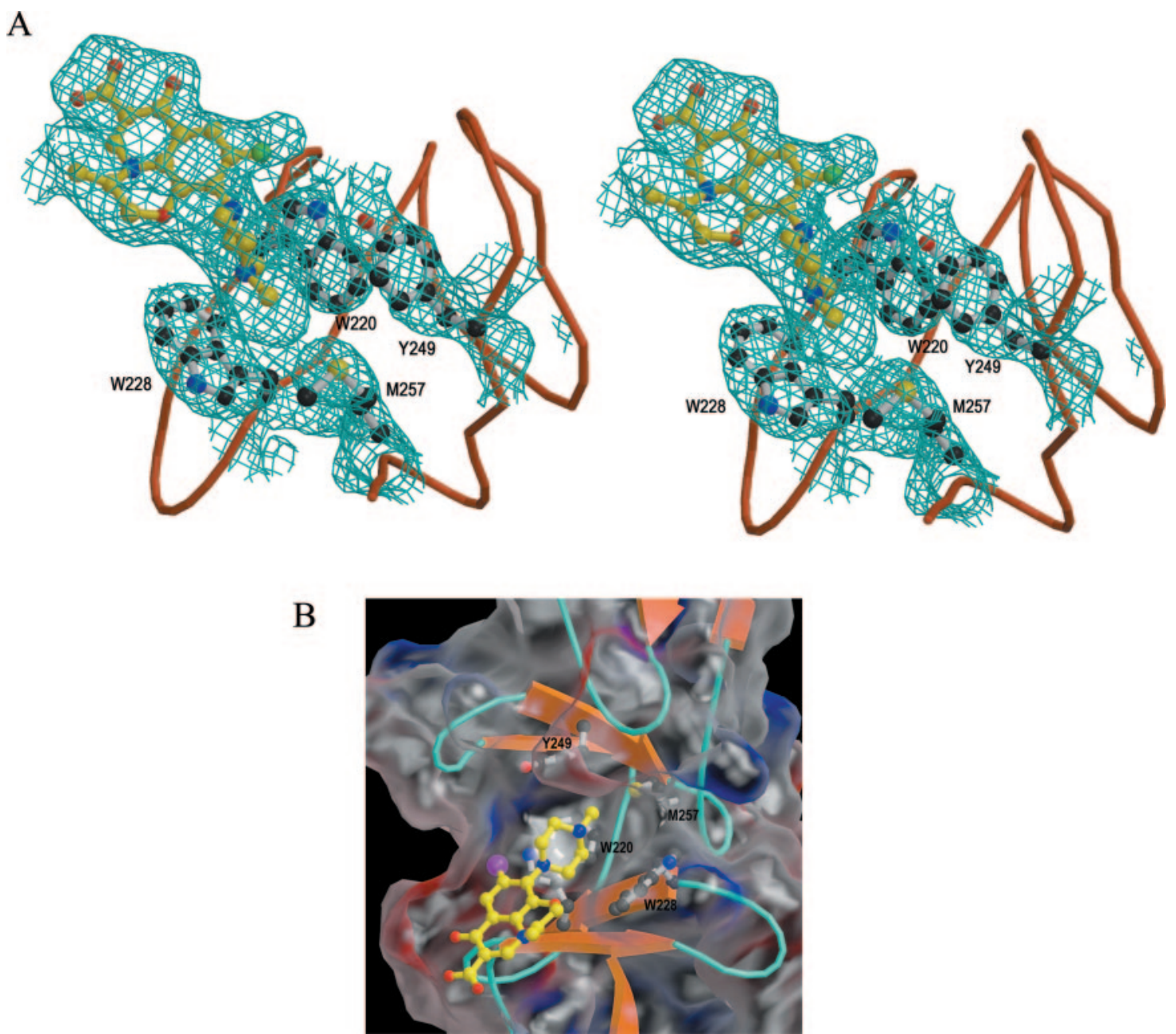

FIG. 3. Crystal structure of dextrofloxacin bound to a ChBS of C-LytA. $A$, stereo view of the $2 F_{o}-F_{c}$ map of dextrofloxacin in the ChBS of C-LytA, contoured at $1 \sigma$. Density is well defined for the dextrofloxacin molecule. All the molecules have been represented as ball and stick. Yellow, red, blue, and green have been used to color $\mathrm{C}, \mathrm{O}, \mathrm{N}$, and $\mathrm{F}$ atoms, respectively. $B$, a solid surface representation of the ChBS with bound dextrofloxacin. The hydrophobic residues and the dextrofloxacin molecule are represented as ball and stick, with $\mathrm{C}, \mathrm{O}, \mathrm{N}$, and $\mathrm{F}$ atoms colored yellow, red, blue, and magenta, respectively. The electrostatic surface potential contoured from -15 (intense red) to +15 kiloteslas (intense blue). The figure was generated with GRASP (45).

ofloxacin. However, it appears to be clear that neither the interaction of norfloxacin nor lomefloxacin with the ChBD of LytA stabilizes the three-dimensional structure in the same way as choline and ofloxacin. Further studies with ChBPs that do not irreversibly denature in the absence of choline (39) may help to resolve this dilemma.

Fluoroquinolone antimicrobial agents were introduced in the 1980s and show considerable bactericidal activity against a broad spectrum of Gram-positive and Gram-negative microorganisms, including $S$. pneumoniae $(40,41)$. Their targets are, preferentially, DNA gyrase in the case of Gram-negative and topoisomerase IV among the Gram-positive bacteria $(42,43)$. The development of these antibacterial agents was the result of intense research aimed at improving the stability, decreasing the affinity and broadening the activity spectrum of the lead compounds 7-chloroquinoline and nalidixic acid. A piperazine group that occupies the choline site in the three-dimensional structure shown here was introduced to extend the half-life of these antimicrobial agents at a certain stage of development. However, the experiments described here suggest that piperazine, when attached to a bulky group, may constitute a new avenue to develop inhibitors of pneumococcal virulence, targeting factors such as the autolytic enzyme LytA. Moreover, the data suggest that this sort of compound may have the same effect on other pneumococcal cell wall-degrading enzymes like
LytC, which has been shown to be critical for the nasopharyngeal colonization stage of infection by this bacterium (5). The inhibition of totally different ChBPs (LytA, LytC, and Cpl-1) by the compounds that displace choline, like the ofloxacin-like compounds reported here, confirms that the ChBDs from different proteins have a similar architecture. Furthermore, this range of activity also suggests that this sort of compound may be used to inhibit the whole family of pneumococcal (and other Gram-positive bacteria) virulent microorganisms, which have virulence factors belonging to the ChBP family (44). Finally, because choline-containing teichoic acids are essential for pneumococcal viability in vivo, the appearance of mutant strains resistant to these new ChBS-targeted antimicrobial agents should be a rare event.

The future pharmacological relevance of the lead compounds outlined in the above will depend on how much their affinity for ChBDs can be boosted. The diversity in the inhibition of LytA by different piperazine-containing quinolones suggests that chemical alteration of this ring to modify affinity is indeed a possibility. Indeed, the crystal structure and the inhibition data presented here may provide a basis for future developments in such a direction. Moreover, the results shown here combined with studies aimed to establish the molecular basis of the role of the ChBDs in the conversion of certain ChBPs from the inactive to the active form and of the putative denaturation 

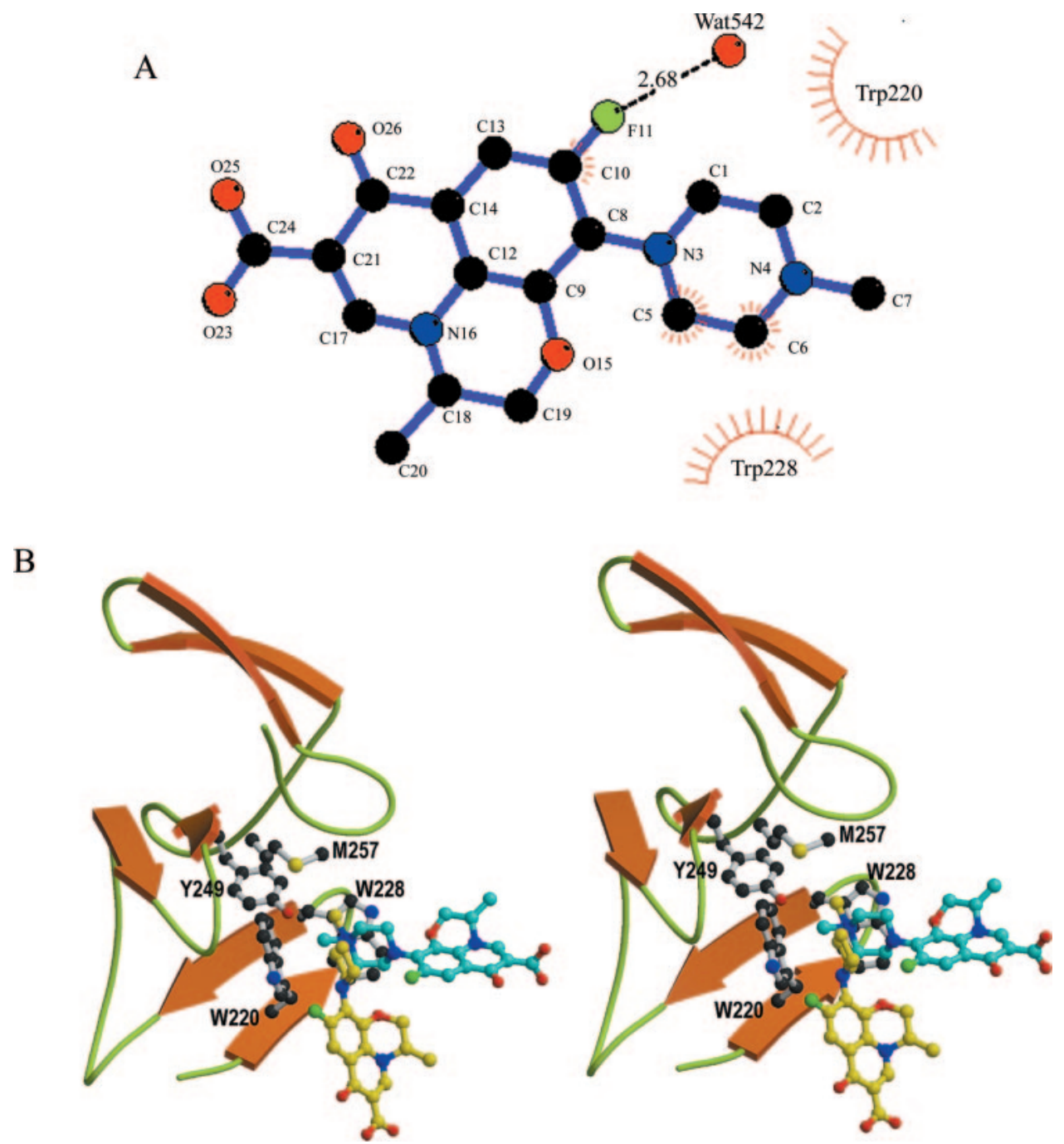

FIG. 4. Geometry of dextrofloxacin at the ChBS in C-LytA. A, diagram of the interactions of dextrofloxacin with C-LytA. Carbon atoms are colored black, oxygens are red, and nitrogens are blue. Hydrogen bonds are symbolized by broken lines. Spiked atoms correspond to those involved in hydrophobic contacts, and spiked circle segments surround hydrophobic van der Waals partners. This figure was drawn with LIGPLOT (7). B, stereo view of the superimposition of the dextrofloxacin molecule at the ChBS as observed in the crystal structure (yellow) and modeled with AutoDock (cyan).

TABLE V

Inhibition of the enzymatic activity of four different lytic enzymes of the pneumococcal system by selected ofloxacin-like compounds

\begin{tabular}{lccccc}
\hline & \multicolumn{5}{c}{$\mathrm{IC}_{50}(\mathrm{~mm})^{b}$} \\
\cline { 2 - 6 } Enzyme $^{b}$ & Choline & Ofloxacin & Norfloxacin & Lomefloxacin & $\begin{array}{c}\text { Pipemidic } \\
\text { acid }\end{array}$ \\
\hline LytA & 25 & 20 & 2 & 2 & 20 \\
LytC & 20 & 2.5 & $\mathrm{ND}^{c}$ & ND & ND \\
Cpl-1 & 2 & 0.3 & ND & ND & ND \\
Cpl-7 & $>175^{d}$ & $>50^{d}$ & ND & ND & ND \\
\hline
\end{tabular}

${ }^{a}$ Pure enzyme preparations were used except in the case of the non-converted LytA where crude $E$. coli extracts were used.

${ }^{b}$ Fifty percent inhibitory concentration. Compounds were dissolved at $150 \mathrm{~mm}$ in $0.2 \mathrm{~m} \mathrm{NaOH}$ except lomefloxacin and nalidixic acid, which were dissolved at $100 \mathrm{~mm}$ in $0.2 \mathrm{~mm} \mathrm{NaOH}$ and distilled water, respectively.

${ }^{c} \mathrm{ND}$, not determined.

${ }^{d}$ Highest concentration tested.

of LytA by norfloxacin, lomefloxacin, and pipemidic acid might also open additional pathways for the development of novel drugs against very important bacterial diseases.

Acknowledgments-We thank the staff of beamline BM14-S at European Synchrotron Radiation Facility (Grenoble, France) for support. We are grateful to C. Fernández-Cabrera and E. Cano for excellent technical assistance.

\section{REFERENCES}

1. Hausdorff, W. P., Bryant, J., Paradiso, P. R., and Siber, G. R. (2000) Clin. Infect. Dis. 30, 100-121

2. Hollingshead, S. K., and Briles, D. E. (2001) Curr. Opin. Microbiol. 4, 71-77 3. McCormick, A. W., Whitney, C. G., Farley, M. M., Lynfield, R., Harrison, L. H., Bennett, N. M., Schaffner, W., Reingold, A., Hadler, J., Cieslak, P., Samore, M. H., and Lipsitch, M. (2003) Nat. Med. 9, 424-430

4. Jedrzejas, M. J. (2001) Microbiol. Mol. Biol. Rev. 65, 187-207

5. Gosink, K. K., Mann, E. R., Guglielmo, C., Tuomanen, E. I., and Masure, H. R. (2000) Infect. Immun. 68, 5690-5695

6. García, J. L., Sánchez-Beato, A. R., Medrano, F. J., and López, R. (2000) in Streptococcus pneumoniae. Molecular Biology and Mechanisms of Disease (Tomasz, A., ed) pp. 231-244, Mary Ann Liebert, Larchmont, NY

7. Wallace, A. C., Laskowski, R. A., and Thornton, J. M. (1995) Protein Eng. 8, 127-134

8. Fischer, W. (2000) in Streptococcus pneumoniae. Molecular Biology and Mechanisms of Disease (Tomasz, A., ed) pp. 155-177, Ann Mary Liebert, Inc., Larchmont, NY

9. López, R., and García, E. (2004) FEMS Microbiol. Rev. 28, 553-580

10. de las Rivas, B., García, J. L., López, R., and García, P. (2002) J. Bacteriol. 184, $4988-5000$

11. García, P. González, M. P. García, E., García, J. L., and López, R. (1999) Mol. Microbiol. 33, 128-138

12. Tuomanen, E. (1999) Curr. Opin. Microbiol. 2, 35-39

13. Briese, T., and Hakenbeck, R. (1985) Eur. J. Biochem. 146, 417-427

14. García, E., García, J. L., Ronda, C., García, P., and López, R. (1985) Mol. Gen. Genet 201, 225-230

15. Giudicelli, S., and Tomasz, A. (1984) J. Bacteriol. 158, 1188-1190

16. Höltje, J.-V., and Tomasz, A. (1975) J. Biol. Chem. 250, 6072-6076

17. Fernández-Tornero, C., López, R., García, E., Giménez-Gallego, G., and Romero, A. (2001) Nat. Struct. Biol. 8, 1020-1024

18. Fernández-Tornero, C., García, E., López, R., Giménez-Gallego, G., and Romero, A. (2002) J. Mol. Biol. 321, 163-173 
19. López, R., García, E., García, P., and García, J. L. (2004) in The Pneumococcus (Tuonamen, E., ed) pp. 75-88, American Society for Microbiology, Washington, D. C.

20. García, J. L., García, E., and López, R. (1987) Arch. Microbiol. 149, 52-56

21. García, J. L., García, E., Arrarás, A., García, P., Ronda, C., and López, R. (1987) J. Virol. 61, 2573-2580

22. Díaz, E., López, R., and García, J. L. (1991) J. Biol. Chem. 266, 5464-5471

23. Sánchez-Puelles, J. M., Sanz, J. M., García, J. L., and García, E. (1992) Eur. J. Biochem. 203, 153-159

24. Sanz, J. M., López, R., and García, J. L. (1988) FEBS Lett. 232, 308-312

25. Morris, G. M., Goodsell, D. S., Halliday, R. S., Huey, R., Hart, W. E., Belew, R. K., and Olson, A. J. (1998) J. Comput. Chem. 19, 1639-1662

26. Leslie, A. G. (1999) Acta Crystallogr. D 55, 1696-1702

27. CCP4. (1994) Acta Crystallogr. Sect. D 50, 760-763

28. Navaza, J. (1994) Acta Crystallogr. A 50, 157-163

29. Brünger, A. T., Adams, P. D., Clore, G. M., DeLano, W. L., Gros, P., GrosseKunstleve, R. W., Jiang, J. S., Kuszewski, J., Nilges, M., Pannu, N. S., Read, R. J., Rice, L. M., Simonson, T., and Warren, G. L. (1998) Acta Crystallogr. Sect. D 54, 905-921

30. Laskowski, R. A., MacArthur, M. W., Moss, D. S., and Thornton, J. M. (1993) J. Appl. Crystallogr. 26, 283-291

31. Tummino, P. J., Ferguson, D., Jacobs, C. M., Tait, B., Hupe, L., Lunney, E., and Hupe, D. (1995) Arch. Biochem. Biophys. 316, 523-528
32. Sotriffer, C. A., W. Flader, W., Winger, R. H., Rode, B. M., Liedl, K. R., and Varga, J. M. (2000) Methods 20, 280-291

33. Voigt, J. H., B. Bienfait, B., Wang, S., and Nicklaus, M. C. (2001) J. Chem. Inf Comput. Sci. 41, 702-712

34. Roussel, A., and Cambilleau, C. (1991) Turbo-Frodo (Directory, P., ed) pp. 77-79, Silicon Graphics, Mountain View, CA

35. García, P., García, J. L., García, E., Sánchez-Puelles, J. M., and López, R. (1990) Gene (Amst.) 86, 81-88

36. Berry, A. M., and Paton, J. C. (2000) Infect. Immun. 68, 133-140

37. Shoichet, B. K., McGovern, S. L., Wei, B., and Irwin, J. (2002) Curr. Opin. Chem. Biol. 6, 439-446

38. Jorgensen, W. L. (2004) Science 303, 1813-1818

39. Romero, P., López, R., and García, E. (2004) J. Bacteriol. 186, 8229-8239

40. Sahm, D. F., Peterson, D. E., Critchley, I. A., and Thornsberry, C. (2000) Antimicrob. Agents Chemother. 44, 2521-2524

41. Chen, D. K., McGeer, A., de Azavedo, J. C., and Low, D. E. (1999) N. Engl. J. Med. 341, 233-239

42. Paton, J. H., and Reeves, D. S. (1988) Drugs 36, 193-228

43. Hooper, D. C. (2001) Clin. Infect. Dis. 32, Suppl.1, S9-S15

44. Wren, B. W. (1991) Mol. Microbiol. 5, 797-803

45. Nicholls, A., Bharadwaj, R., and Honig, B. (1993) Biophys. J. 64, 166 (Abstr. Tu-Pos189) 


\section{Ofloxacin-like Antibiotics Inhibit Pneumococcal Cell Wall-degrading Virulence}

Factors

Carlos Fernández-Tornero, Ernesto García, Beatriz de Pascual-Teresa, Rubens López, Guillermo Giménez-Gallego and Antonio Romero

J. Biol. Chem. 2005, 280:19948-19957.

doi: 10.1074/jbc.M501236200 originally published online March 15, 2005

Access the most updated version of this article at doi: 10.1074/jbc.M501236200

Alerts:

- When this article is cited

- When a correction for this article is posted

Click here to choose from all of JBC's e-mail alerts

This article cites 40 references, 13 of which can be accessed free at http://www.jbc.org/content/280/20/19948.full.html\#ref-list-1 